\title{
Optimization of Multi-standard Transmitter Architecture Using Single-Double Conversion Technique Used for Rescue Operations
}

\author{
Riadh Essaadali ${ }^{1}$, Said Aliouane ${ }^{2}$, Chokri Jebali ${ }^{2}$ and Ammar Kouki ${ }^{2}$ \\ ${ }^{1}$ Aviat Networks, H9H3L4, Canada \\ ${ }^{2}$ LACIME, Electrical engineering department, École de technologie supérieure, H3C1K3, Canada
}

\author{
A R T I C L E IN F O \\ Article history: \\ Received: 06 March, 2017 \\ Accepted: 29 March, 2017 \\ Online: 09 April, 2017 \\ Keywords : \\ Multi-standard, transmitter, \\ optimization, direct conversion, \\ single-double conversion, UAV \\ and rescue operation
}

\begin{abstract}
A B S T R A C T
A new architecture of multi-standard transmitter that combines single and double frequency conversion is presented. The transmitter is able to generate waveforms of different cellular standards (GSM, UMTS, Wimax), LAN standards (Bluetooth, Zigbee, IEEE802.11 b/g) and Radio navigation standards (GPS, Galileo) as well. Depending on the selected standard and frequency, the transmitter routes the intermediate frequency signal, into direct or double conversion path. Optimization design techniques are introduced to manage multiple standards and efficiently enable them while reducing cost, size and weight of the resulting transmitter. Such a transmitter is aimed to be used as a backup solution for a broken network to reestablish and insure communication links for rescue operations.
\end{abstract}

\section{Introduction}

On January 12th 2010, an earthquake has devastated Haiti, causing approximatively 160.000 deaths [1]. One of the main reasons that worsen the disaster was some major failures in the wireless communications towers, located at Port-au-Prince, which hampered the emergency measures.

Disasters have no specific target location and no specific timing. One of the possible solutions to insure the communication between rescue and intervention units is to deploy Unmanned Aerial Vehicles (UAVs, a.k.a Drones) to restore the links with the wearable transmitters in case of emergency and to generate radio navigation signals when satellite signals are lost. As illustrated in Figure 1, an UAV equipped with a multi-standard transceiver will fly over the affected city to take over the communication system.

Existing mobile networks are heterogeneous and people own different devices supporting cellular standards (GSM, UMTS and Wimax), wireless personal and local area network standards

*Riadh Essaadali Aviat Networks and LACIME, 16847 Hymus Boulevard, Kirkland, Quebec, H9H3L4,

Email:Riadh.essaadali@aviatnet.com

www.astesj.com

https://dx.doi.org/10.25046/aj020311
(Bluetooth, Zigbee and IEEE802.11 b/g) as well as Radio navigation (GPS and Galileo). In this context, the UAV's transceivers have to support all available wireless communication standards and all operating frequencies. Therefore, there is a real need to a multi-standard transceiver that covers common wireless standards and automatically switches between different standards and frequencies whenever needed.

Some solutions have been already proposed. Indeed, Qualcomm transmitter in [2] serves different GSM variants (GSM850, Primary GSM, EDGE, Railway GSM, and GSM1800 \& GSM1900). In [3], another architecture is proposed to generate GSM signals using Phased Locked Loops (PLLs) in the upconverters eliminating the additional filters traditionally used between the up-converter and the amplification bloc. This solution minimizes power consumption but is only applicable to constant envelope signals. Another architecture [4] was proposed for both constant and complex envelope signals. Each waveform is generated by a different configuration of I/Q modulators.

The proposed solution involves a new architecture that generates the RF signal using direct or double conversion, depending on the desired standard. In addition, an optimization 


\section{R. Essaadali et. al. / Advances in Science, Technology and Engineering Systems Journal Vol. 2, No. 3, 73-81 (2017)}

technique is introduced to minimize the high number of RF filters and PLLs necessary to cover multiple standards and frequencies. Therefore, cost, size and weight are also minimized.

This paper is an extension of work originally presented in IEEE MILCOM2016 [5] and it is organized as follows: Section II outlines the basics of multistandard architecture optimization. The judicious filtering technique is detailed. After presenting the frequency plan of different standards to be served, two architectures with their different components will be presented. In the last section, both of architectures are validated by carrying out simulations with CAD software: Advanced Design System (ADS).

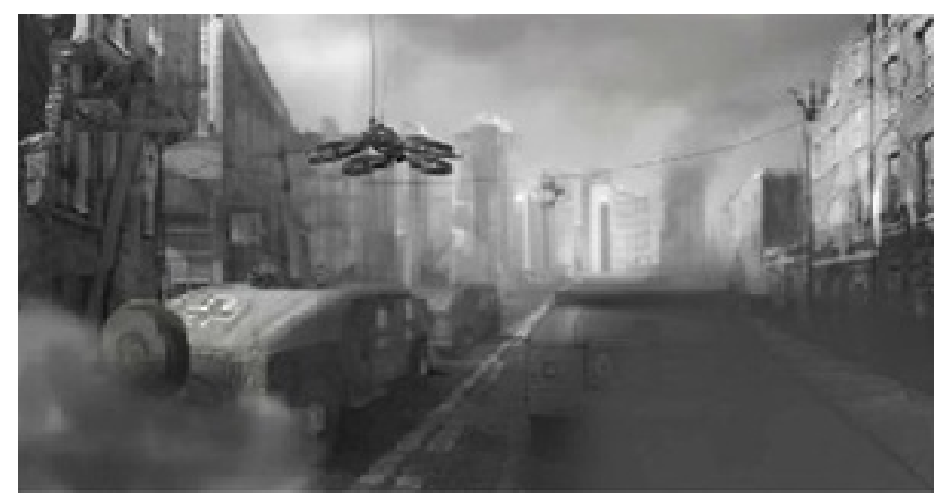

Figure 1 Artist point of view of a rescue operation using UAVs [1]

\section{Multi-standard transmitter architecture definition and optimization}

\subsection{Basics of architecture optimization}

The first step in the transmitter design is defining the architecture. Generally, the transmitter is divided into the following blocks: baseband part, frequency up-converter, RF filter, power amplifier and antenna. Modern architectures could be heterodyne, homodyne, polar and digital-IF. The digital IF which is heterodyne is the most used because of its perfect balance between I and Q signals. Its architecture is illustrated in Figure 2. Digital I and Q signals are combined in the baseband part. The resulting signal is converted by a digital to analog converter.

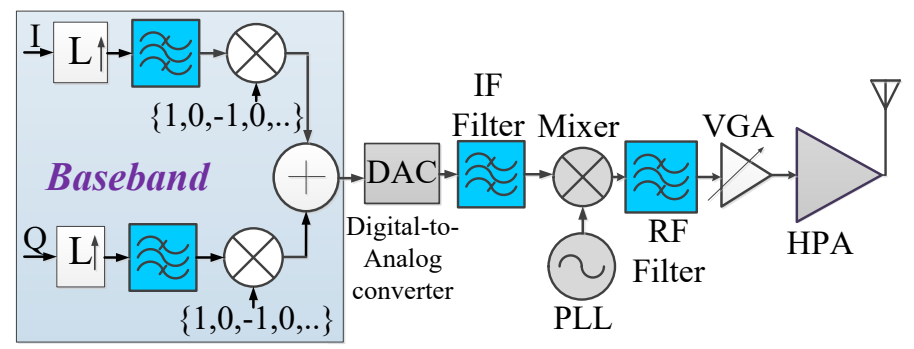

Figure 2 Architecture of digital IF (heterodyne) transmitter

The frequency $f_{\mathrm{IF}}$ of the signal at the IF stage is then upconverted to $f_{\mathrm{RF}}$, at the output of the up-converter. In order to reduce the number of required filters, in a multi-standard transmitter, the upper side band signal located in $f_{\mathrm{RF}}=f_{\mathrm{LO}}+f_{\mathrm{IF}}$ and the lower side band signal located in $f_{\mathrm{RF}}=f_{\mathrm{LO}}-f_{\mathrm{IF}}$ are used. The mixing of the IF signal and the carrier produces the desired output signal, the image signal and the intermodulation products. As illustrated in Figure 3, the desired signal can be located either on the upper or lower sideband. Harmonics and inter-modulation products have the form $\mathrm{m} * f_{\mathrm{LO}}+\mathrm{n} * f_{\mathrm{IF}}$, and the critical harmonics are: $-f_{\mathrm{LO}}+5 f_{\mathrm{IF}}, f_{\mathrm{LO}}-3 f_{\mathrm{IF}}, f_{\mathrm{IF}}, f_{\mathrm{LO}}, f_{\mathrm{LO}}+3 f_{\mathrm{IF}}, f_{\mathrm{LO}}+5 f_{\mathrm{IF}}$. The IF-frequency $\mathrm{f}_{\mathrm{IF}}$ is fixed whereas the PLL-frequency $\mathrm{f}_{\mathrm{LO}}$ is tuned. If the signal is transmitted in the upper sideband, the filer should reject the nearest unwanted signal components $f_{\mathrm{LO}}$ and $f_{\mathrm{LO}}+3 f_{\mathrm{IF}}$. In the same way, if the signal is transmitted in the lower sideband, the rejection of $f_{\mathrm{LO}}$ and $f_{\mathrm{LO}}-3 f_{\mathrm{IF}}$ is required. This concept allows increasing the bandwidth of the RF filters, and therefore, it reduces their numbers.

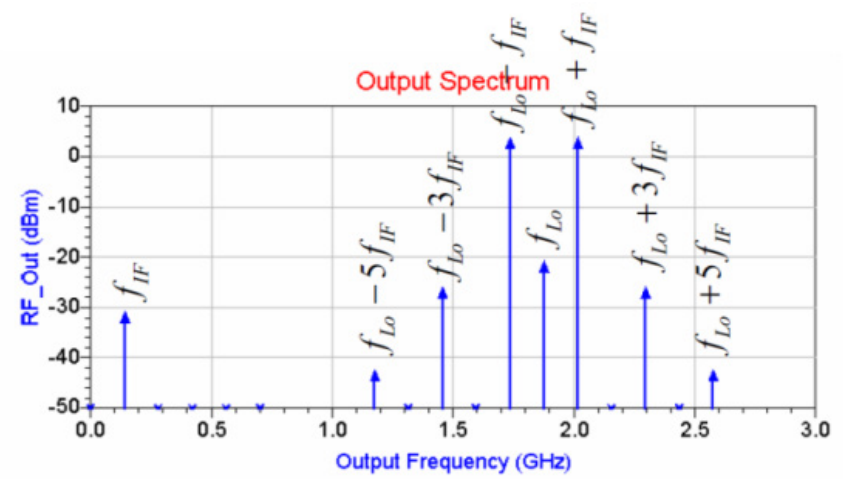

Figure 3 A typical output spectrum of the up-converter

Moreover, it decreases the frequency range that should be covered by the PLLs. Indeed, the sub-frequency band of the PLL that will cover the higher sub-band of the filter will be re-used to cover the lower sub-band of the filter. Figure 4 illustrates this concept. In conventional direct-conversion architecture, the maximum stop bandwidth of a filter is lower than $f_{\mathrm{IF}}$. By using the optimization concept, the stop bandwidth of each RF filter is lower than $2 f_{\mathrm{IF}}$. An overview of this filtering strategy is given in Figure 5. The difference between the upside $\left(f_{\text {StopHigh }}\right)$ and low side ( $\left.f_{\text {StopLow }}\right)$ stop band frequencies of the RF filter should be at most equal to $2 f_{\mathrm{IF}}$. Two scenarios are included in Figure 5 , the higher lateral band $\left(f_{\mathrm{LO} 1}+f_{\mathrm{IF}}\right)$ is filtered where $f_{\mathrm{LO} 1}$ is the frequency generated by the local oscillator. In the second scenario, the lower lateral band $\left(f_{\mathrm{LO} 2}-f_{\mathrm{IF}}\right)$ is filtered by the same filter where $f_{\mathrm{LO} 2}$ is the frequency generated by the local oscillator.

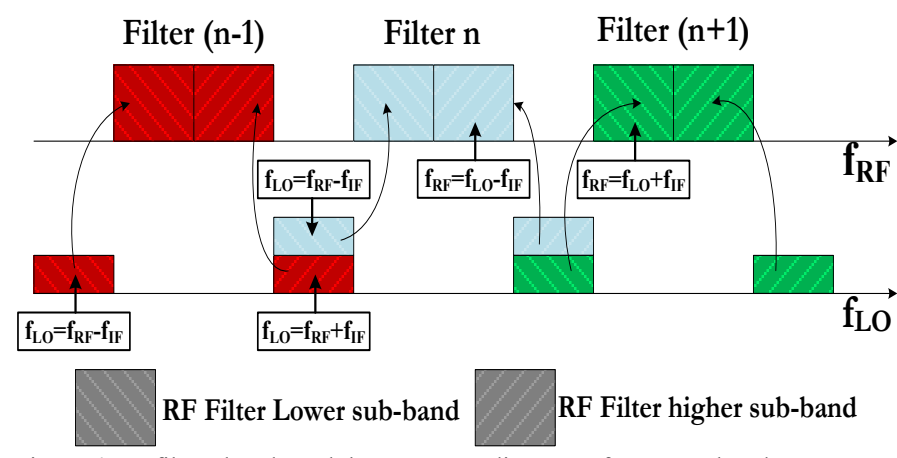

Figure 4 RF filters bands and the corresponding PLL frequency bands. 


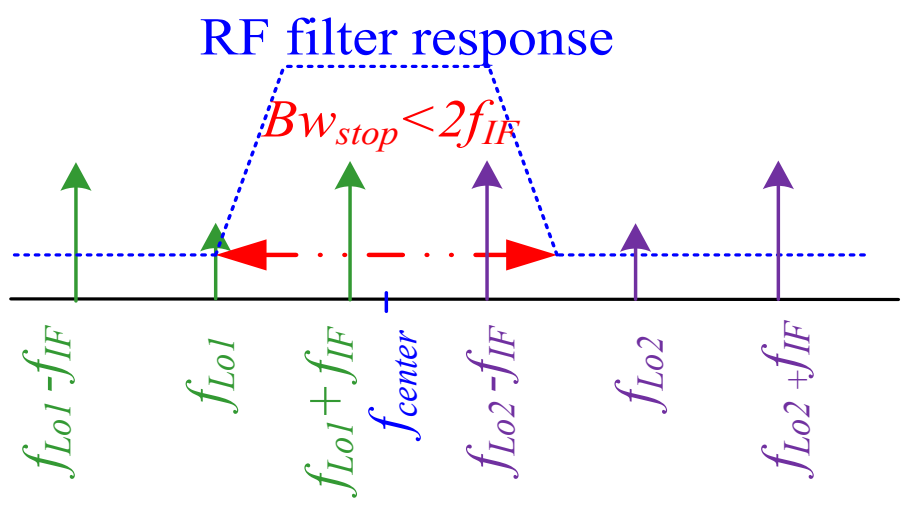

Figure 5 Judicious filtering technique.

\subsection{Optimization of a Direct Conversion Multi-standard Transmitter}

The frequency plan to be covered is shown in Figure 6. The second generation of a cellular communication standard (GSM) offers voice and low speed data transfer [6]. The need to add multimedia services at a very high-speed in wireless communication infrastructure has led to the development of the third generation such as UMTS and the fourth generation as Wimax [7]. Local and Personal wireless networks such as WLAN, Bluetooth and Zigbee have become very popular [8]. And satellite navigation systems were developed to enable precise and reliable positions for users. European Union launched a new positioning system called Galileo and United States of America have upgraded their satellite navigation system [9], [10].

The method consists on subdividing the whole frequency band into sub-bands, respecting the fact that the rejection bandwidth does not exceed $2 f_{\text {IF }}$. Another point that should be considered is that closer the pass band is to $2 f_{\mathrm{IF}}$, the steeper the slope of the filter transfer function has to be. Therefore, the order of the filter increases and the number of resonators increases as well which increases the size of the RF filters. The rejection bandwidth should not exceed $2 f_{\mathrm{IF}}$. Thus, in order to reduce the number of required RF filters, The IF frequency $f_{\mathrm{IF}}$ should be high enough.

The maximum frequency that the afforded digital to analog converter can generate is $140 \mathrm{MHz}$. Thus, the transmitter requires 8 RF filters, each one having a bandwidth less than 2 fIF $=2 \times 140=280 \mathrm{MHz}$. the standards served by each RF filter are shown in Figure 7. Filter 1 covers the standards GSM, UMTS and Zigbee between $824 \mathrm{MHz}$ and $960 \mathrm{MHz}$. Filter 2 covers GPS and Galileo signals. It has a bandwidth of $136 \mathrm{MHz}$ centered at 1232 MHz. Filter 3 covers also GPS and Galileo signals between 1559 $\mathrm{MHz}$ and $1575 \mathrm{MHz}$. Filter 4 covers the standards GSM and UMTS between $1710 \mathrm{MHz}$ and $1785 \mathrm{MHz}$. Filter 5 covers also the standards GSM and UMTS between $1805 \mathrm{MHz}$ and $1990 \mathrm{MHz}$. Filter 6 covers the UMTS standard between $2010 \mathrm{MHz}$ and 2170 MHz. Filter 7 covers Wimax, Bluetooth, Zigbee and WLAN standards between $2300 \mathrm{MHz}$ and $2497 \mathrm{MHz}$. Filter 8 covers UMTS and Wimax standards between $2496 \mathrm{MHz}$ and $2690 \mathrm{MHz}$.

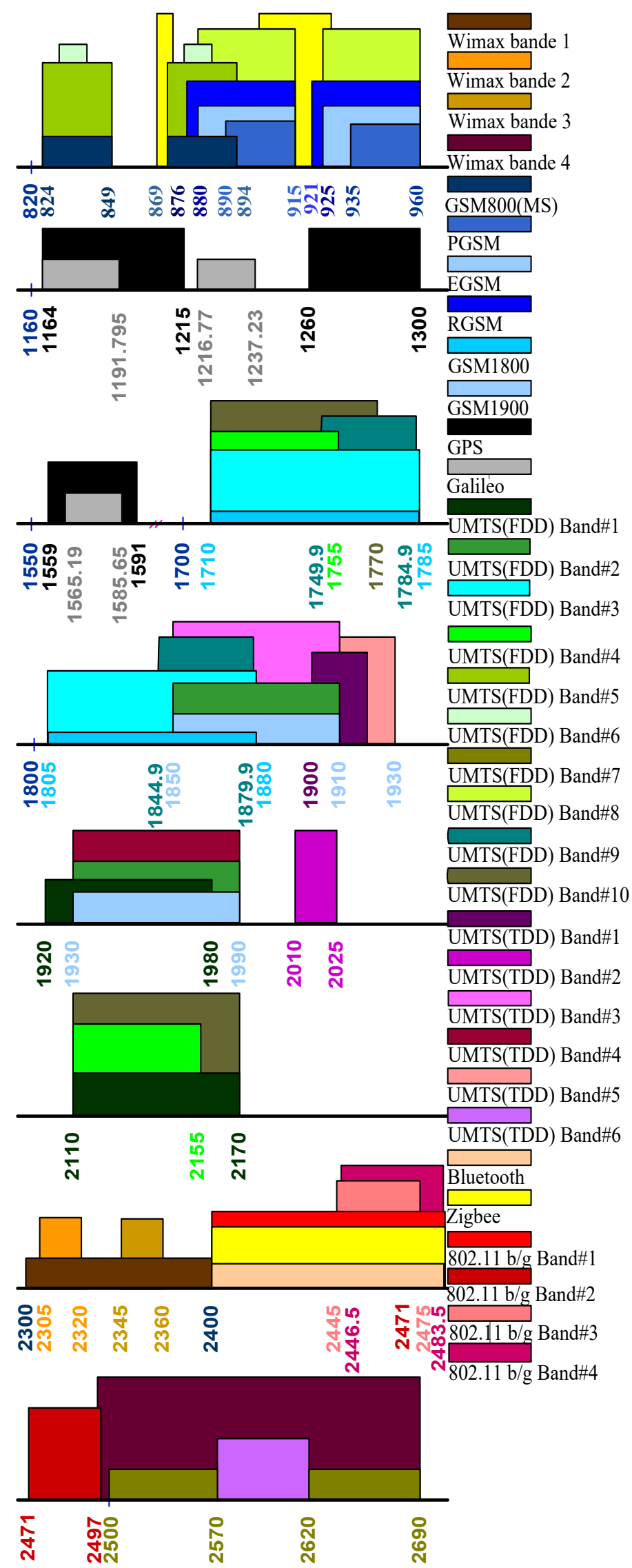

Figure 6 Frequency plan of the considered standards 


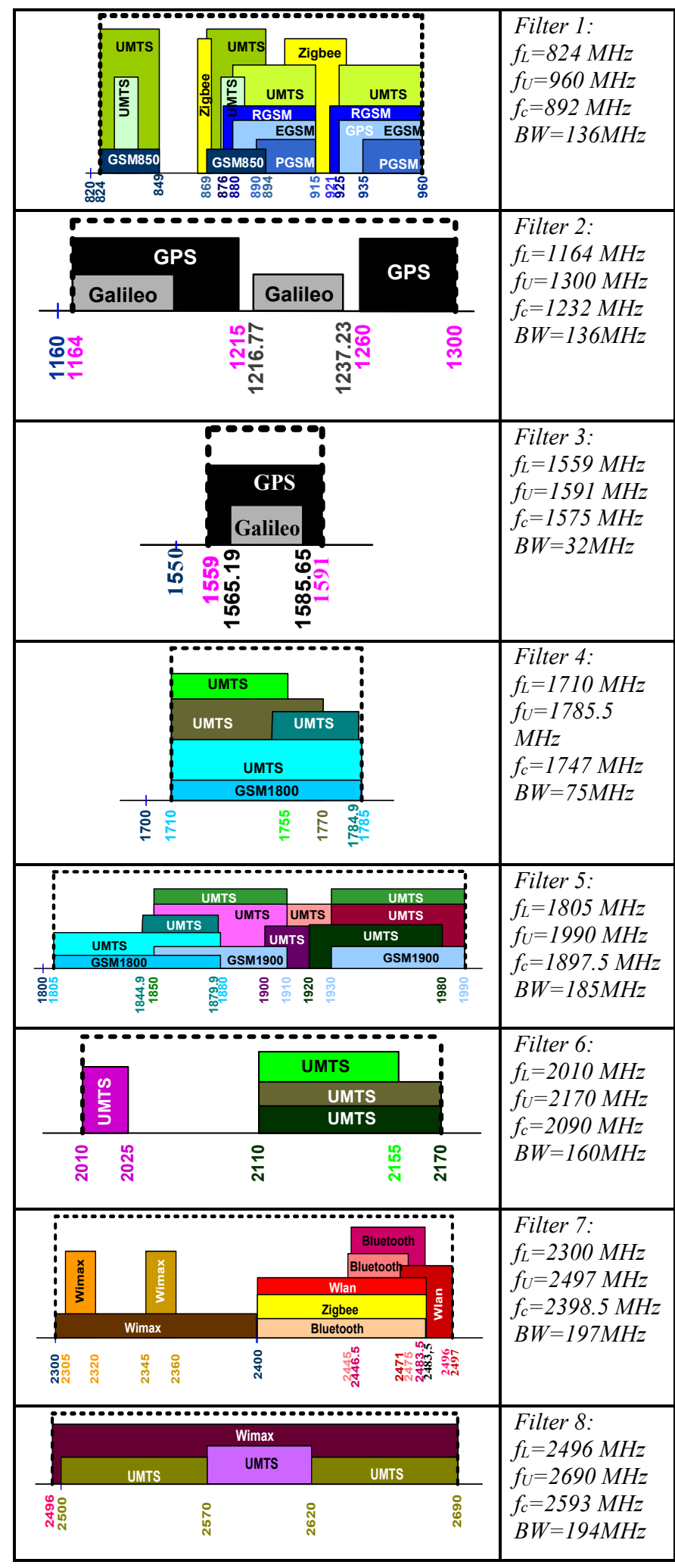

Figure 7 RF filters specifications.

Figure 8 shows how the multi-standard architecture generates and processes the right signal. GPS signal is taken here as an example to illustrate the process. At the DAC output the FPGA generate the GPS signal that passes through an IF filter of $32 \mathrm{MHz}$ bandwidth, centered at $140 \mathrm{MHz}$. This corresponds to the maximum pass band allowed by the whole set of standards. The filtered signal is then up-converted to the desired RF frequency www.astesi.com using a Phase Locked Loop (PLL). A combination of a two digitally controlled SPDT and SP4T switches insures the routing of the signal to the GPS filtering path which is selected among 8 RF filtering arms. Finally, the filtered signal is amplified before transmission.

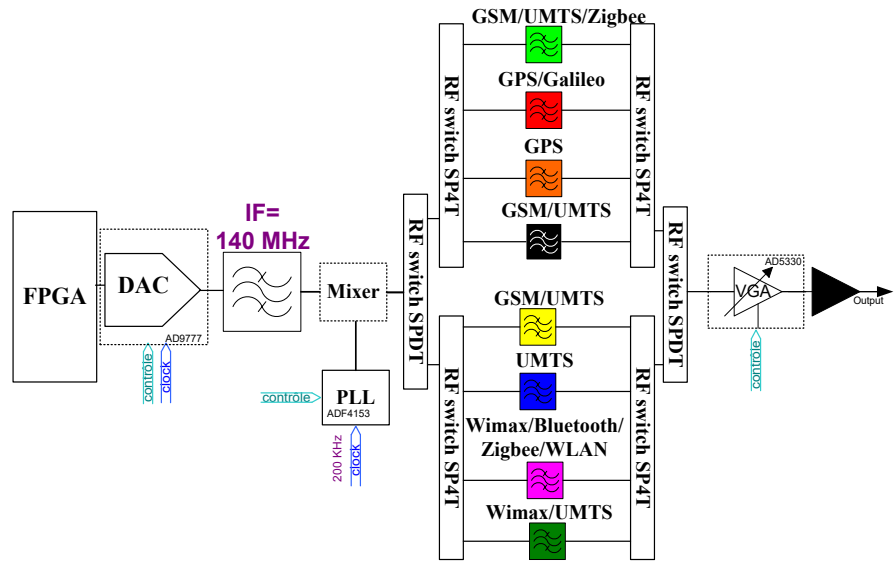

Figure 8 The proposed multi-standard transmitter architecture.

The frequency band covered by the voltage controlled oscillator used in the PLL depends on RF filter bandwidths. For a given filter having a central frequency $f_{c}$ and a bandwidth $B W$, the frequency range is:

$$
\begin{array}{lll}
{\left[f_{\mathrm{c}}-B W / 2-f_{\mathrm{IF}} ; f_{\mathrm{c}}-f_{\mathrm{IF}}\right]} & \text { if we choose } & f_{\mathrm{RF}}=f_{\mathrm{IF}}+f_{\mathrm{LO}},(1) \\
{\left[f_{\mathrm{c}}+f_{\mathrm{IF}} ; f_{\mathrm{c}}+f_{\mathrm{IF}}+B W / 2\right]} & \text { if we choose } & f_{\mathrm{RF}}=f_{\mathrm{LO}}-f_{\mathrm{IFF}} .
\end{array}
$$

Figure 9 shows the obtained frequency ranges to be covered by the frequency synthesizer.

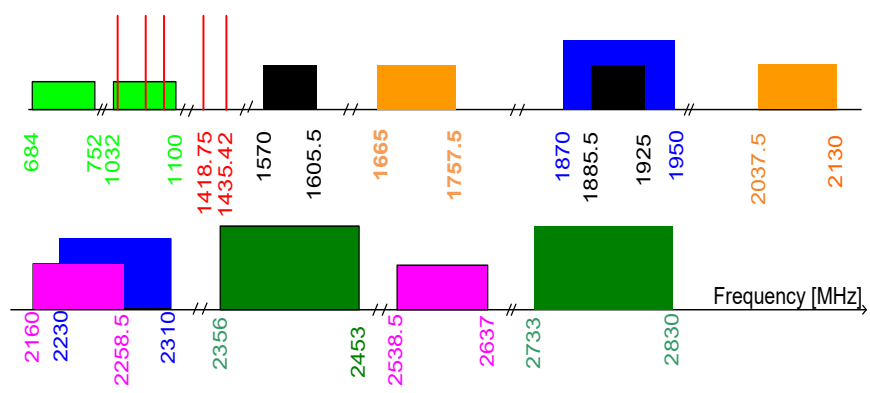

Figure 9 Frequency bands to be covered by the frequency synthesizer.

The mixer is selected primarily depending on its frequency band operation. The characteristics of the mixer are shown in Table 1. The frequency band of the LO port has to cover 824 and 2690 MHz. It has to exhibit a high IIP3 and a high $1 \mathrm{~dB}$ compression point, as well as low insertion loss. Regarding those conditions, the best candidate is MACOM's ESMD-C50M mixer.

For the SPDT switch, many device candidates are possible. The Analog Devices' ADG918 exhibits the lowest insertion loss and return loss and offers the highest isolation and IIP3. For SP4T, 


\section{R. Essaadali et. al. / Advances in Science, Technology and Engineering Systems Journal Vol. 2, No. 3, 73-81 (2017)}

the Analog Devices'ADG904 fulfil the requirements. The specifications of the RF switches are presented in Table 2.

Table 1 Mixer main characteristics

\begin{tabular}{|c|c|}
\hline Mixer Name & MACOM ESMD-C50M \\
\hline RF band $[\mathrm{MHz}]$ & $10-2950$ \\
\hline IF band $[\mathrm{MHz}]$ & $30-590$ \\
\hline LO band $[\mathrm{MHz}]$ & $10-2950$ \\
\hline Conversion Loss $[\mathrm{dB}]$ & 6.21 \\
\hline LO drive level $[\mathrm{dBm}]$ & 13 \\
\hline
\end{tabular}

Table 2 SPDT \& SP4T main characteristics

\begin{tabular}{|c|c|c|}
\hline Name & $\begin{array}{c}\text { SPDT- } \\
\text { ADG918 }\end{array}$ & $\begin{array}{c}\text { SP4T- } \\
\text { ADG904 }\end{array}$ \\
\hline Freq band [MHz] & DC - 4000 & DC-2700 \\
\hline Insertion Loss [dB] & 0.8 & 1.1 \\
\hline Isolation [dB] & 43 & 37 \\
\hline Retun Loss [dB] & 26 & 27 \\
\hline $\mathrm{IP}_{1 \mathrm{~dB}}[\mathrm{dBm}]$ & 17 & 16 \\
\hline $\mathrm{IIP}_{3}[\mathrm{dBm}]$ & 36 & 31 \\
\hline
\end{tabular}

The IF filter has to be centered at $140 \mathrm{MHz}$ with a minimum bandwidth of $32 \mathrm{MHz}$. Low ripple, low insertion loss and low group delay are required. High stop band rejection and return loss are also needed. Oscilent's 820 -IF140.0M is selected as it satisfies all the requirements. Its characteristics are presented in Table 3.

Table 3 IF Filter characteristics

\begin{tabular}{|c|c|}
\hline Name & Oscilent820-IF140.0M \\
\hline Center Freq [MHz] & 140 \\
\hline Bandwidh $[\mathrm{MHz}]$ & 34 \\
\hline Ripple $(\mathrm{dB})$ & 0.65 \\
\hline Group delay (us) & 0.96 \\
\hline Rejection $(\mathrm{dB})$ & 60 \\
\hline Insertion Loss $(\mathrm{dB})$ & 22.2 \\
\hline
\end{tabular}

The RF filter reponse depends on the characteristics of the chosen RF components, especially the frequency conversion bloc. The rejection level required to attenuate the level of $f_{\mathrm{LO}}, f_{\mathrm{LO}} \pm 3 f_{\mathrm{IF}}$ and of the other remaining intermodulation products should be determined to respect the out-of-band emission mask. A software simulation (ADS) is used to obtain the level of different harmonics and intermodulation products at the input of RF filters and at the output of the first stage of RF switches.

The common RF filter response is plotted in Figure 10. The attenuation $A_{\min }$ should be low in the filter passband between $\mathrm{F}_{\mathrm{c}^{-}}$ $F_{\mathrm{p}}$ and $F_{\mathrm{c}}+F_{\mathrm{p}}$. The term $A_{f_{L o}}$ is the attenuation level needed to reject the oscillator signal. The frequency bands between $F_{c}-F_{p^{-}}$ $f_{\mathrm{IF}}$ and $F_{c^{-}} f_{\mathrm{IF}}$ and between $F_{c}+f_{\mathrm{IF}}$ and $F_{c}+F_{p}+f_{\mathrm{IF}}$ correspond to the frequency range of the PLL frequency $f_{\mathrm{LO}}$. The term $\mathrm{A}_{\max }$ is the attenuation level to be achieved for the image frequency. The frequency bands between $F_{c}-F_{p}-2 f_{\mathrm{IF}}$ and $F_{c}-2 f_{\mathrm{IF}}$ and between
$F_{c}+2 f_{\mathrm{IF}}$ and $F_{c}+F_{p}+2 f_{\mathrm{IF}}$ correspond to the frequency range of the image signal. The term $A_{f_{L o} \pm 3 f_{I F}}$ is the minimum level required to attenuate the intermodulation product located in $f_{L o}-3 f_{I F}$ and $f_{L o}+3 f_{I F}$. The frequency bands between $F_{c}-F_{p}-4 f_{\mathrm{IF}}$ and $F_{c^{-}}-4 f_{\mathrm{IF}}$ and between $F_{c}+4 f_{\mathrm{IF}}$ and $F_{c}+F_{p}+4 f_{\mathrm{IF}}$ correspond to the frequency range of the harmonic components $f_{L o}-3 f_{I F}$ and $f_{L o}+3 f_{I F}$ respectively. The term $A_{f_{L o} \pm 5 f_{I F}}$ is the minimum level required to attenuate the harmonics located in $f_{L o}-5 f_{I F}$ and $f_{L o}+5 f_{I F}$. The frequency bands between $F_{c^{-}} F_{p^{-}}-6 f_{I F}$ and $F_{c^{-}}-6 f_{I F}$ and between $F_{c}+6 f_{I F}$ and $F_{c}+F_{p}+6 f_{I F}$ correspond to the frequency range of the harmonic components $f_{L o}-5 f_{I F}$ and $f_{L o}+5 f_{I F}$ respectively. The term $A_{f_{I F}}$ is the attenuation level of the IF component.

Different standards have an attenuation levels depending on the in- and out-of-band emission limits. The level of each harmonic components and undesired signals at the input of the RF filters are designed with a software simulation. The obtained harmonics and intermodulation products rejection levels for each filter are summarized in Table 4. The RF power amplifier is assumed to be linear to guarantee that the levels of the generated harmonics and intermodulation products generated by the upconverter relative to the signal level are kept to be similar after the amplification bloc.

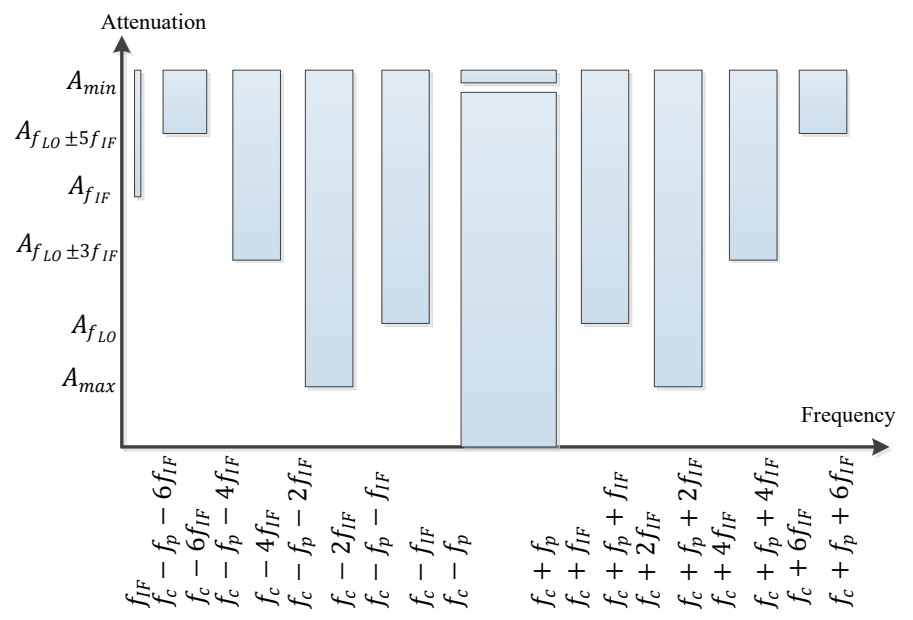

Figure 10 RF Filter response

Table $4 \mathrm{~F}_{\mathrm{LO}}$ and $\mathrm{F}_{\mathrm{LO}} \pm 3 \mathrm{~F}_{\mathrm{IF}}$ rejections for each channel filter

\begin{tabular}{|c|c|c|c|c|}
\hline Specification & $\begin{array}{l}\text { Center } \\
\text { Freq } \\
{[\mathrm{MHz}]}\end{array}$ & $\begin{array}{l}\text { Pass } \\
\text { band } \\
\text { Freq } \\
{[\mathrm{MHz}]}\end{array}$ & $\begin{array}{l}\mathrm{f}_{\mathrm{LO}} \pm 3 \mathrm{f}_{\mathrm{IF}} \\
\text { rejection } \\
\text { level }[\mathrm{dB}]\end{array}$ & $\begin{array}{l}\mathrm{f}_{\mathrm{LO}} \\
\text { rejection } \\
\text { level }[\mathrm{dB}]\end{array}$ \\
\hline Filter 1 & 892 & 136 & 80 & 59 \\
\hline Filter 2 & 1232 & 136 & 40 & 19 \\
\hline Filter 3 & 1575 & 32 & 40 & 19 \\
\hline Filter 4 & 1747.5 & 75 & 80 & 59 \\
\hline Filter 5 & 1897.5 & 185 & 80 & 59 \\
\hline Filter 6 & 2090 & 160 & 53 & 32 \\
\hline Filter 7 & 2398.5 & 197 & 52 & 31 \\
\hline Filter 8 & 2593 & 194 & 53 & 32 \\
\hline
\end{tabular}




\section{R. Essaadali et. al. / Advances in Science, Technology and Engineering Systems Journal Vol. 2, No. 3, 73-81 (2017)}

\subsection{Optimization of a Multi-standard transmitter architecture using single/double conversion}

The first architecture uses eight RF filters, two SPDT switches, four SP4T switches. This architecture is costly in terms of size. For optimization purposes, we have developed another architecture based on double conversion. Two stages are used to transpose the signal from an intermediate frequency to RF frequency.

In order to reduce further the number of required RF filters, The IF frequency fIF should be sufficiently high. The maximum frequency that the afforded DAC can generate is $140 \mathrm{MHz}$. The purpose is to cover the maximum standards with the minimum number of filters. The judicious filtering technique detailed in the section II.A is re-used to develop this architecture.

The first part of the spectrum is $824-1300 \mathrm{MHz}$. For this section of the spectrum, the direct conversion method with an IF frequency of $140 \mathrm{MHz}$ (which leads to a maximum channel filter bandwidth of $280 \mathrm{MHz}$ ) gives good results in terms of rejections. However, the second section of the spectrum $(1559-2690 \mathrm{MHz})$ requires at least six IF-filters to be entirely covered. To reduce the total size and cost of this previous setting, we use instead, a double conversion with a new IF-frequency that will be high enough to require at most two band-pass filters without being too close to the LO frequency. This prevents to generate harmonic interference.

In fact, the intermediate frequency is not fixed for this architecture, it is chosen in such a way that it does not exceed the third of the frequency of the phase-locked loop, $f_{L O} / 3$. Indeed, at the output of each mixer at both frequency conversion blocs, the critical harmonics that should be filtered are $-f_{L o}+5 f_{I F}, f_{I F}$, $f_{L o}-3 f_{I F}, f_{L o}, f_{L o}+3 f_{I F}, f_{L o}+5 f_{I F}$. These harmonics may disturb the judicious filtering technique when one of them, at least, falls within the frequency ranges between $f_{L o}-2 f_{I F}$ and $f_{L o}+2 f_{I F}$. The RF filter bandwidth is at most equal to twice the intermediate frequency

$$
\begin{gathered}
\max \left(f_{I F}\right)=\frac{2}{7} f_{L O}=\frac{2}{9} f_{R F} \\
\Delta f \leq 2 * \min \left(f_{I F}\right)
\end{gathered}
$$

Looking at Figure 6, the frequency bands of interest start at $f_{R F}=824 \mathrm{MHz}$. According to (3), the maximum IF frequency is therefore $206 \mathrm{MHz}$. In one hand, if the carriers around $824 \mathrm{MHz}$ are generated by double conversion, the maximum of the frequency at the output of the digital converter must be $51.5 \mathrm{MHz}$. In the other hand, if the signal is generated by direct conversion, the maximum of the intermediate frequency is $140 \mathrm{MHz}$ and the first filter covers the frequency band between $824 \mathrm{MHz}$ and 960 $\mathrm{MHz}$. The bandwidth of the filter is $136 \mathrm{MHz}$ and the minimum intermediate frequency $f_{I F}$ is $68 \mathrm{MHz}$. The first filter bandwidth covers GSM, UMTS and Zigbee standards. The second filter starts at $f_{R F}=1164 \mathrm{MHz}$. According to (3), the maximum IF frequency $f_{I F}$ is therefore $291 \mathrm{MHz}$ in the case of direct conversion. The bandwidth of the filter is $136 \mathrm{MHz}$ therefore the minimum frequency is $68 \mathrm{MHz}$. The frequency bands to be covered which come just after the second filter start at frequency $1559 \mathrm{MHz}$. The corresponding maximum IF frequency is $389.75 \mathrm{MHz}$. The third filter covers the band 1559-1990 MHz. The maximum frequency and minimum frequency is therefore $215.5 \mathrm{MHz}$. The fourth filter covers the frequency band between $2010 \mathrm{MHz}$ and $2690 \mathrm{MHz}$. The maximum intermediate frequency is $502.5 \mathrm{MHz}$ and the minimum intermediate frequency is $340 \mathrm{MHz}$. Simulations using Advanced Design System (ADS) of Keysight technologies on this set up (see Simulation section) prove that the second stage IF frequency of 357.5 $\mathrm{MHz}$ is a good compromise and corresponds to a central frequency of an available intermediate filter. The maximum allowed channel filter bandwidth is $715 \mathrm{MHz}$. The filters 1 and 2 are used for the direct conversion whereas the filters 3 and 4 are used for the double conversion. Specifications of each RF filter are summarized in Figure 11.

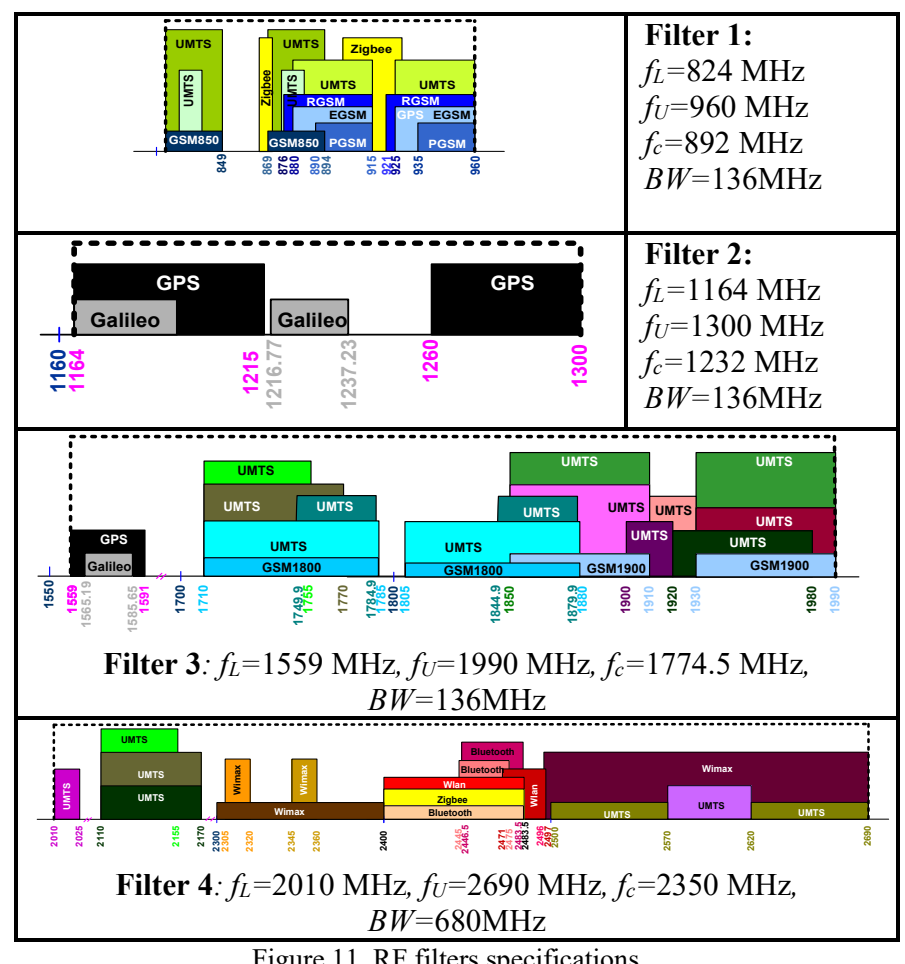

Figure 11 RF filters specifications.

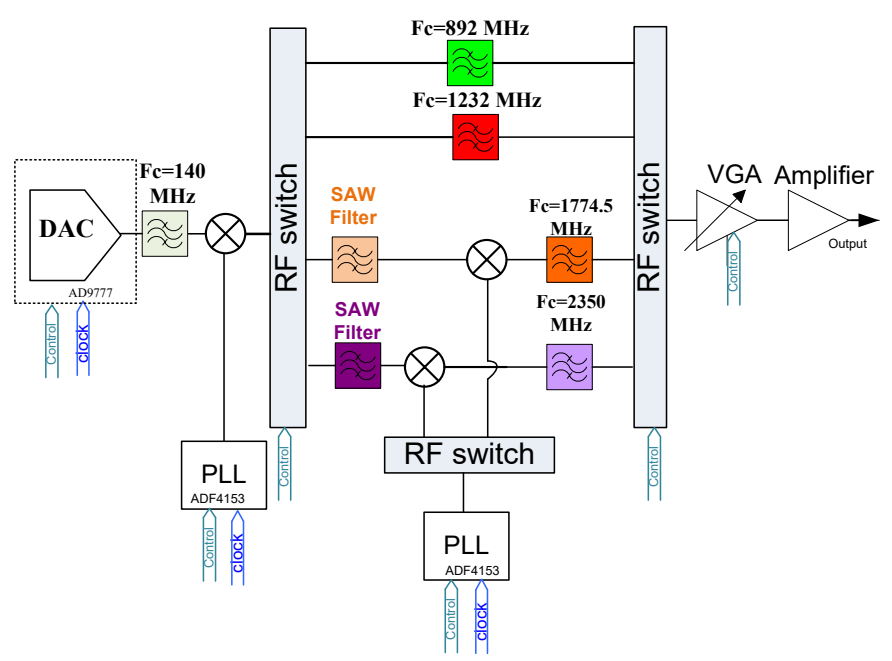

Figure 12 The proposed multi-standard transmitter architecture.

The global transmitter architecture is shown in Figure 12. The DAC output is filtered by an IF-filter with a $32 \mathrm{MHz}$ bandwidth (corresponding to the maximum pass band of the GPS standards) centered at $140 \mathrm{MHz}$. The signal is then up-converted to a desired 


\section{R. Essaadali et. al. / Advances in Science, Technology and Engineering Systems Journal Vol. 2, No. 3, 73-81 (2017)}

RF frequency. Then, depending on the RF frequency, the signal is routed to either single or double conversion path. For the double conversion, two intermediate surface acoustic wave (SAW) filters centered at $357.5 \mathrm{MHz}$ and having at least $32 \mathrm{MHz}$ bandwidth are to be considered before the second up-conversion stage. The signal is then driven by a variable gain amplifier (VGA) depending on the standard and finally amplified by a high power amplifier (PA).

Figure 13 shows the obtained frequency for the local oscillators. The green bands of $684-752 \mathrm{MHz}$ and $1032-1100 \mathrm{MHz}$ are the LO frequencies corresponding to the direct conversion path (filters 1 and 2). For double conversion path we use the up sideband frequency $497.5 \mathrm{MHz}(357.5+140)$ because it is closer to the other required bands. The orange and purple bands corresponds to the LO frequencies of the second PLL for the $3^{\text {rd }}$ and $4^{\text {th }}$ filters respectively (low side band and higher side band).

In the frequency synthesizer's side, the frequency range to be covered by the Voltage-Controlled Oscillator (VCO) depends on the bandwidth of the filters. For a filter whose center frequency $f_{c}$ is and the bandwidth is $B_{W}$, the frequency range to be covered by the VCO is between $f_{c}-B_{W} / 2-f_{I F}$ and $f_{c}-f_{I F}$ on the one hand and between $f_{c}+f_{I F}$ and $f_{c}+B_{W} / 2+f_{I F}$ on the other hand. Figure 13 shows the total frequency range to be covered by the RF frequency synthesizers. For the signals covered by the first two filters covering the frequency bands $824-960 \mathrm{MHz}$ and $1164-1300 \mathrm{MHz}$ respectively, the intermediate frequency $f_{I F}$ is set to $140 \mathrm{MHz}$. In the path where the frequency transposition occurs twice, the intermediate frequency at the output of the analog-digital converter is fixed and is equal to 140 $\mathrm{MHz}$. The signal generated by the converter is transposed to 357.5 $\mathrm{MHz}$ by the first block of the frequency synthesizer. The first frequency synthesizer should generate a frequency of $497.5 \mathrm{MHz}$ or $217.5 \mathrm{MHz}$. The frequency $497.5 \mathrm{MHz}$ is chosen because it is closer to the other frequency ranges. The second block of the frequency synthesizer serves to transpose the $357.5 \mathrm{MHz}$ frequency signal to a RF signal that will be filtered by the third and fourth filters.

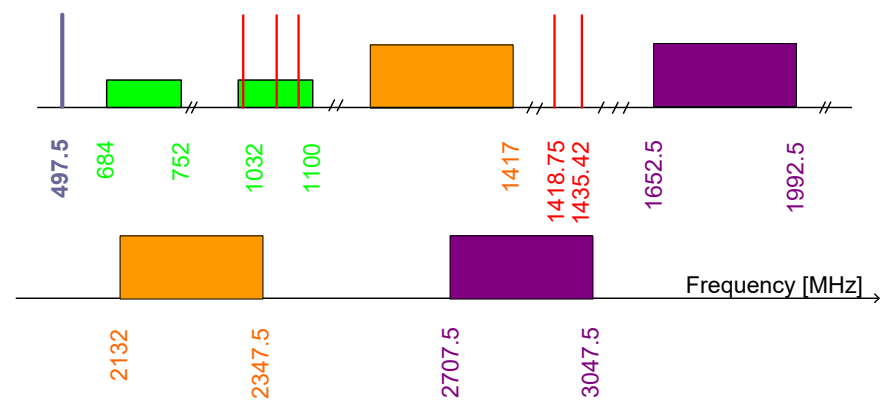

Figure 13 Frequency bands to be covered by the frequency synthesizer

Between the two mixers, a filter should be inserted. In the market, SAW (Surface Acoustic Wave) filters can be used. The third filter covers GPS, Galileo, UMTS, GSM and WLAN standards with a maximum channel width of $32 \mathrm{MHz}$. The fourth filter covers the UMTS, WIMAX, Bluetooth, Zigbee and WLAN standards with a maximum bandwidth of $28 \mathrm{MHz}$. The first and the second SAW are pass-band filters. The center frequency of the first SAW is located between $215.5 \mathrm{MHz}$ and $389.75 \mathrm{MHz}$ and between $340 \mathrm{MHz}$ and $502.5 \mathrm{MHz}$, for the second. Their bandwidth should be at least $32 \mathrm{MHz}$ and $20 \mathrm{MHz}$ respectively. Figure 14 illustrates the possible locations of IF channels to be filtered by the third and fourth filters, i.e, at the second frequency conversion bloc.

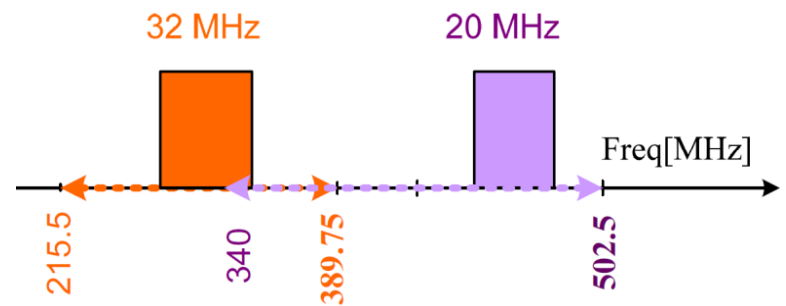

Figure 14 Possible frequencies of intermediate IF channels.

The folding of the two intermediate frequency ranges is considered as an optimization point if a filter that covers the frequency band between $389.75 \mathrm{MHz}$ and $340 \mathrm{MHz}$ is available. From the market, the chosen SAW filter is RFM SF2090C as its ripple, group delay and insertion loss are low and its out-of-band rejection is high. Its characteristics are in Table 5.

The modified transmitter architecture is illustrated in Figure 15. The transmitter includes an analog to digital converter, a mixer, phase locked loops, an SPDT type RF switch, two SP4T type RF switches, a variable gain amplifier and an RF amplifier block. The chosen mixer is MACOM's ESMD-C50M. Its main characteristics are listed in TABLE I. The RF switches ADG918 and ADG904 of analog devices are the best candidates. Their characteristics are in TABLE II. After the DAC, the IF filter (Oscilent's 820-IF140.0M) is inserted. Its characteristics are listed in Table 1.

Table 5 SAW IF filter characteristics

\begin{tabular}{|c|c|}
\hline SAW filter Name & RFM SF2090C \\
\hline Center freq [MHz] & 357.5 \\
\hline Bandwidth [MHz] & 35 \\
\hline Ripple [dB] & 1 \\
\hline Group delay [ns] & 55 \\
\hline Rejec-tion [dB] & 40 \\
\hline Insertion Loss [dB] & 12 \\
\hline Frequency Band [MHz] & $340-375$ \\
\hline
\end{tabular}

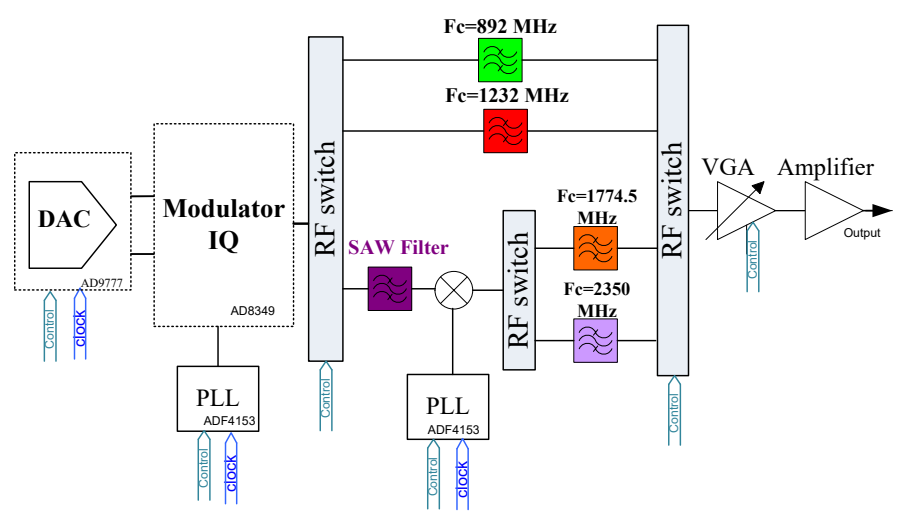

Figure 15 The architecture of single/dual multi-standard transmitter. 


\section{R. Essaadali et. al. / Advances in Science, Technology and Engineering Systems Journal Vol. 2, No. 3, 73-81 (2017)}

The out-of-band attenuations of the first second filters are determined based on the level of the harmonics at the output of the first mixer and the values of the center frequency and bandwidth. The center frequency of the first filter is $892 \mathrm{MHz}$, and its bandwidth is $136 \mathrm{MHz}$. The first filter covers GSM, UMTS and Zigbee standards. According to their various masks and spurious emissions cited, the rejection of the image frequency should be 80 $\mathrm{dB}$ and of the local oscillator should be $19 \mathrm{~dB}$. The second filter covers the GPS and Galileo systems, the rejection of the image frequency should be $40 \mathrm{~dB}$ and of the component is $19 \mathrm{~dB}$. For the third filter and the fourth filter, the levels of harmonics are obtained by carrying out a harmonic balance simulation. The level of harmonic components $f_{L o}+f_{I F}$ and $f_{L o}-f_{I F}$ is $10.79 \mathrm{dBm}$. The level of the local oscillator signal is -19.64 . According to the Mask signal and out-of-band emission limits of GSM, UMTS, GPS and Galileo, the third filter should attenuate the image signal by $80 \mathrm{~dB}$ and the local oscillator component by $71.15 \mathrm{~dB}$. The stop band of this filter is $720 \mathrm{MHz}$. The fourth RF filter has a bandwidth of $680 \mathrm{MHz}$. It covers UMTS, Bluetooth, Zigbee, WLAN and WIMAX standards. It should attenuate the signal at $f_{\text {Lo }}$ by 44.15 $\mathrm{dB}$ and the image frequency by $53 \mathrm{~dB}$.

\section{Simulation}

\subsection{Simulation of the Direct Conversion Multi-standard Transmitter}

To validate the concept, the multi-standard architecture is implemented in ADS, as shown in Figure 16. The RF filters, the RF switches, the mixer as well as the IF filter are implemented by using their models. The simulation allows checking whether the spurious signals are well rejected by the RF filters. All standards generated through the proposed multi-standard architecture are tested. And for each standard, many frequency step simulation runs were carried out. The output spectrum respects the out of band emission limits, in all simulated cases. The following test run is used as an illustration example.

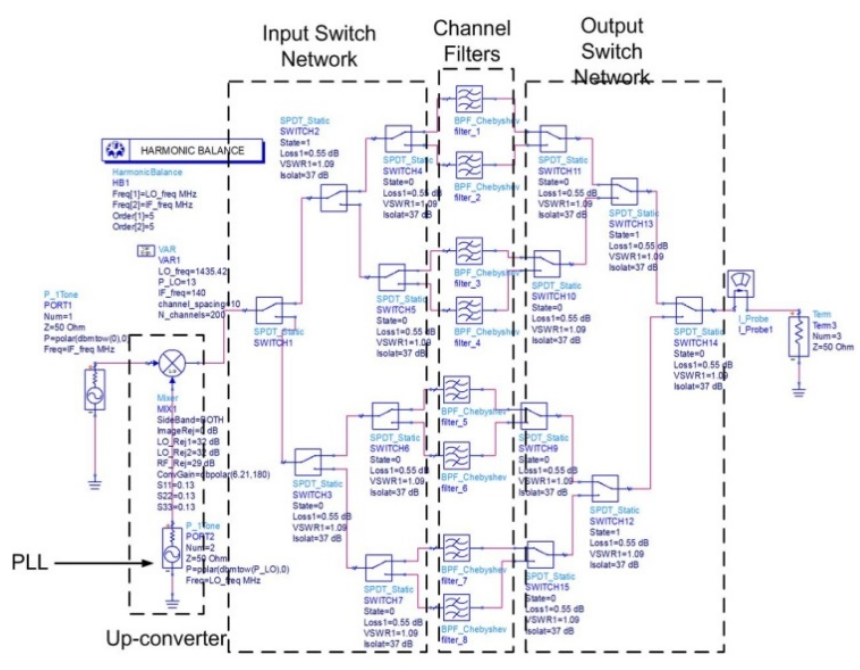

Figure 16 Validation of the direct conversion multi-standard transmitter architecture with ADS.

To generate a WiMAX signal at $2304 \mathrm{MHz}$, the RF switches are activated in a way that the signal is filtered by filter 7 . The frequency of the signal that is generated by the PLL is
$f_{L o}=f_{R F}-f_{I F}=2164 \mathrm{MHz}$. The spectrum of the signal at the output of the transmitter for this case is illustrated in Figure 17.

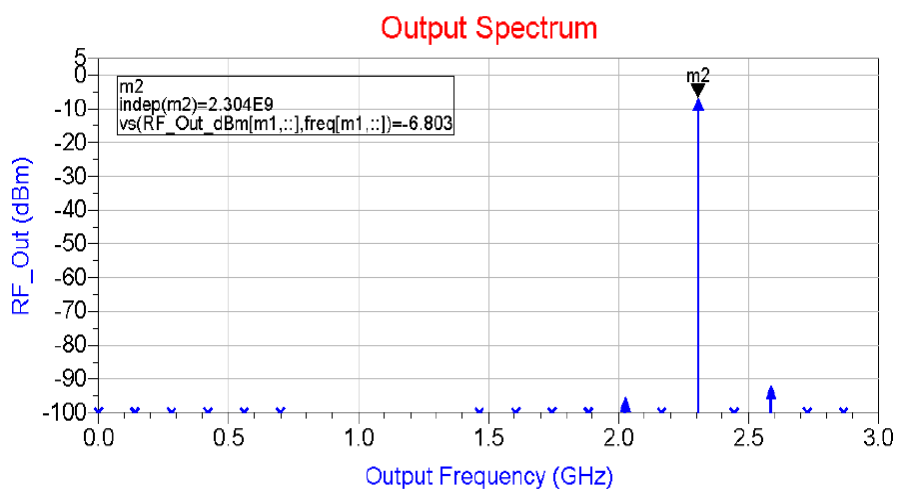

Figure 17 Spectrum of the signal at the output of the transmitter

\subsection{Simulation of a multi-standard transmitter using single/ double conversion technique}

After the selection of the RF components, the simulation of the second architecture is done using the Keysight-ADS simulator. The circuit diagram useful for completing the simulation in ADS is shown in Figure 18. The simulation consists in carrying out the spectral analysis at the output of the transmitter by varying the frequencies of the frequency synthesizers and adjusting the control parameters of the RF switches.

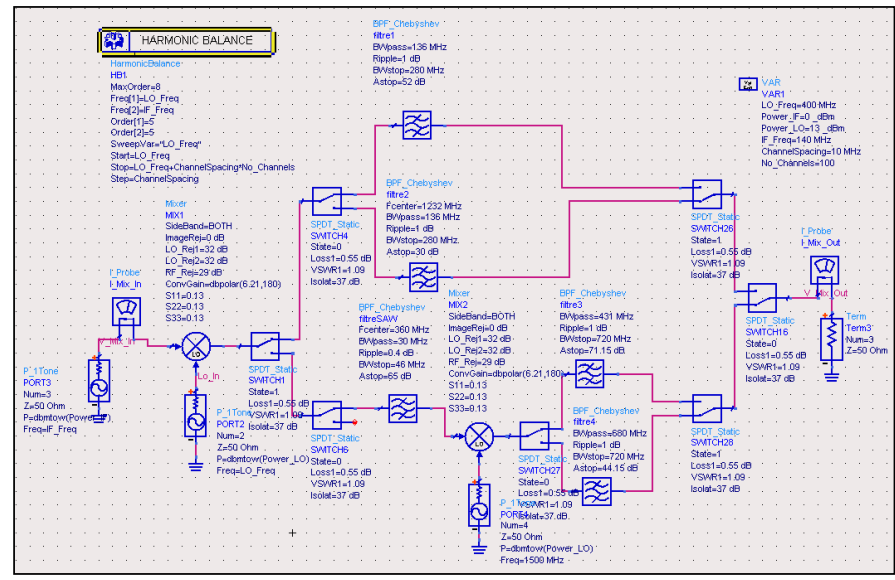

Figure 18 Validation of the multi-standard transmitter architecture using single/double conversion technique with ADS.

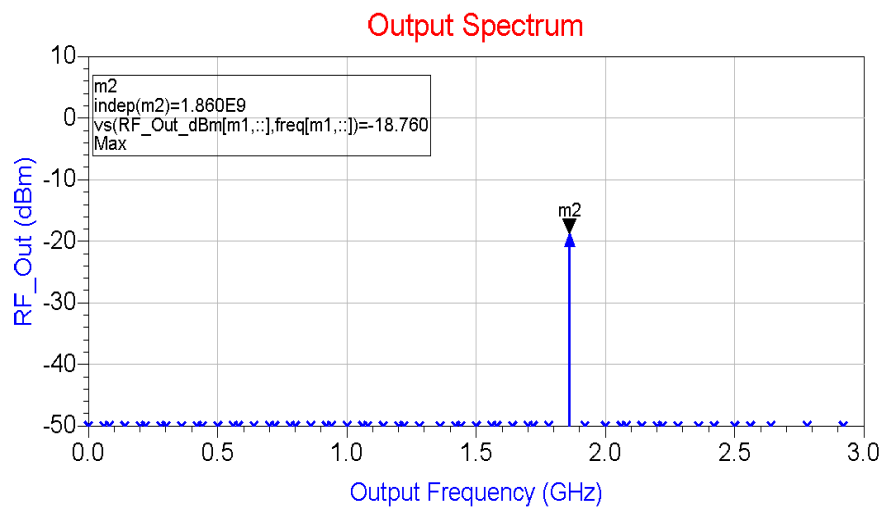

Figure 19 Spectrum of the signal at the output of the transmitter 
For example, if the frequency of the first frequency synthesizer and of the second frequency synthesizer are adjusted to $f_{L o 1}=497.5 \mathrm{MHz}$ and $f_{L o 2}=2217.5 \mathrm{MHz}$. The different RF switches are tuned in a way that the RF signal passes through the third filter. At the output of the transmitter, the spectrum is shown in Figure 19.

\section{Conclusion}

In this paper, two new optimized architectures of a multistandard transmitter have been presented. The first one is an heterodyne based architecture. The second combines single and double frequency conversion that allows further reducing the cost, size and weight of the transmitter. This transmitter is a part of a wireless radio that will be carried by an UAV reaching stricken areas after a disaster and therefore enabling and making easier efficient rescue intervention.

It has been shown, through simulation, that both of architectures can generate GPS and Galileo signals which are very critical for rescue operations and waveforms of different cellular wireless communication standards (UMTS, GSM and Wimax) along with waveforms of different local and personal area wireless network standards (Bluetooth, Zigbee and IEE802.11 b/g). The high number of different wireless communication standards and their operating frequency bands requires a high number of RF filters and PLLs. The first architecture is direct conversion. It uses an analog digital converter to convert the digital signal into an analog signal. The analog signal passes through an IF filter with a center frequency of $140 \mathrm{MHz}$. The filtered signal is mixed with a carrier to be transposed to an RF frequency. The RF signal may be the upper or lower sideband. The RF signal passes through an SP4T switch which points the RF signal to its corresponding RF filter. The first architecture uses eight RF filters. RF filters are partitioned and defined based on the judicious filtering technique. For each RF filter, the lower half of bandwidth serves to filter the upper side signal and the upper half of filter's bandwidth serves to filter the lower side signal. The second architecture is less expensive than the first. It uses fewer components. It uses two SAW IF filters, two mixers, two SP4T RF switches, an SPDT RF switch and four RF filters. The frequency range to be covered by the frequency synthesizers of the second architecture is between 497.5 MHz and 3047.5 MHz. As a logical perspective, the architecture can be further optimized. There is an opportunity to use a frequency doubler that allows using fewer RF filters and fewer RF frequency synthesizers.

\section{References}

[1] F. Gagnon, "La radio cognitive, l'avenir de demain," ed, May 2013.

[2] S. M. Mollenkopf, P. H. A. See, and B. C. Walker, "Multi-standard transmitter system and method for a wireless communication system," ed: Google Patents, 2005.

[3] H. Jensen, "Translational Loop RF transmitter architecture for GSM Radio," United States Patent, vol. US007386283B2, no. Broadcom Corporation, p. 14, Jun. 10,2008 2008 .

[4] J. Kilpatrick, J. Dawson, J. Bohorquez, and J. Venuti, "Programmable transmitter architecture for non-constant and constant envelope modulation," ed: Google Patents, 2007.

[5] R. Essaadali, T. Hadouej, C. Jebali, and A. Kouki, "Optimization of a direct conversion multi-standard transmitter used for rescue operations," in MILCOM 2016 - 2016 IEEE Military Communications Conference, 2016, pp. 1173-1178.
[6] L. Harte and Books $24 \times 7$ Inc. (2005). Introduction to global system for mobile communication (GSM) physical channels, logical channels, network, and operation [Text.].

[7] J. G.Andrews, A. Ghish, and R. Muhamed, Fundamentals of WiMAX Prentice Hall, february 2007.

[8] H. A. H. Labiod, C. De Santis. (Springer, 2007). WI-FI TM, BLUETOOTH TM, ZIGBEE TM AND WIMAX TM.

[9] A. R. CORPORATION, Navstar GPS Space Segment/Navigation User Interfaces, 1993.

[10] J. Kreher, "GALILEO Signals : RF Characteristics," presented at the Navigation System Panel, 2004. 